\title{
Minimal Complexity Sinusoidal Controls for Path Planning
}

\author{
Jean-Paul Gauthier and Matthias Kawski ${ }^{\ddagger}$
}

\begin{abstract}
This article presents simple controls that generate motion in the direction of high order Lie brackets. Whereas the naive use of piecewise constant controls requires the number of switchings to grow exponentially with the length of the bracket, we show that such motion is possible with sinusoidal controls whose sum of frequencies equals the length of the bracket.

This work is closely related and motivated by the study of the complexity of sub-Riemannian geodesics for generic regular distributions, i.e., whose derived flag has maximal growth vector. Of particular interest is the approximation of curves transversal to the distribution by admissible curves.

We also present a surprising example that shows that it is possible to simultaneously kill higher moments without increasing the number of self-intersections of the base curve.
\end{abstract}

\section{INTRODUCTION}

Fundamental to path planning and geometric control is the ability to move in directions of Lie brackets of the control vector fields by switching or oscillating between different control inputs. Motion in the direction of higher order Lie brackets demands more frequent switchings, or higher frequency oscillations. An immediate question is: "How many more switchings?" Writing $e^{t f}$ for the flow of a vector field $f$, i.e., the solution of the differential equation $\dot{x}=f(x)$, a naive algorithm iterates $[X, Y]_{p}(\varphi)=$ $\lim _{t \rightarrow 0} \frac{1}{2 t^{2}}\left(\varphi\left(e^{-t Y} e^{-t X} e^{t Y} e^{t X}(p)\right)-\varphi(p)\right)$ and obtains, e.g., $[[X, Y], Z]$ as a limit involving the composition of flows

$$
e^{-t Z}\left(e^{-t X} e^{-t Y} e^{t X} e^{t Y}\right) e^{t Z}\left(e^{-t Y} e^{-t X} e^{t Y} e^{t X}\right)
$$

This approach leads to an exponentially increasing number of switchings, compare e.g. the example in section 9 of [1]. We prove that it is possible to generate motion in the directions of high order Lie brackets using sinusoidal inputs whose sum of the frequencies is the order of the Lie bracket.

This work is motivated by the recently discovered simple topological structure of the optimal controls (multiple figure eights, topologically equivalent to those pictured in figure 1 ) for path planning problems such as the rolling ball with a trailer [2], [3], [4]. The corresponding control systems may be written in the form

$$
\dot{x}=u_{1} f_{1}(x)+u_{2} f_{2}(x), u_{1}^{2}+u_{2}^{2} \leq 1 .
$$

where the $f_{i}$ are smooth vector fields on an $N$-dimensional manifold $M^{N}$, and $\left(u_{1}, u_{2}\right):[0, T] \mapsto \mathbb{R}^{2}$ is measurable.

Geometrically, the fields $f_{1}$ and $f_{2}$ span a two dimensional distribution $\Delta$. We are interested in generic, maximally

‡ This work was partially supported by the National Science Foundation through the grant DMS 09-08204

Jean-Paul Gauthier is with the LSIS, UMR CNRS 7296, and member of INRIA Team GECO. gauthier@univ-tln.fr

Matthias Kawski is with the School of Mathematical and Statistical Sciences, Arizona State University, Tempe, Arizona 85287, USA, kawski@asu.edu nonintegrable distributions, that is, with maximal growth vector $r=\left(r_{1}, r_{2}, \ldots r_{s-1}, r_{s}\right)=(2,3,5,8, \ldots N-1, N)$ whose components $r_{i}$ are the dimensions of the flag of the distributions defined by $\Delta=\Delta^{[1]}$, and $\Delta^{[k+1]}=\left[\Delta, \Delta^{[k]}\right]$. The constraint $u_{1}^{2}+u_{2}^{2} \leq 1$ corresponds to the declaration that $\left\{f_{1}, f_{2}\right\}$ form an orthonormal basis for $\Delta$, and optimal solutions correspond to sub-Riemannian geodesics [5]. The problem of immediate interest is to approximate an arbitrary curve $\gamma$ in $M^{N}$ that is transversal to $\Delta^{[s-1]}$ by solutions of system (2) that stay within an $\varepsilon$-tube about $\gamma$.

The complexity of a nonadmissible curve $\gamma$ has traditionally been quantified in terms of the lengths of such $\varepsilon$ approximating admissible curves [6], [7], [8]. Technically, one introduces the metric complexity $M C(\varepsilon)$ that asymptotically measures the length of the shortest $\varepsilon$-approximating admissible curves, and the interpolation entropy that measures the length of the shortest admissible curve that interpolates $\gamma$ on intervals of length at most $\varepsilon$. This basically agrees with Kolmogorov's entropy of the curve $\gamma$ for the Carnot-Caratheodory metric defined by the sub-Riemannian structure.

The minimal value of the exponent $(s-1)$ of the complexity is well-studied, [6], [7], [8], [9], [10]. Roughly speaking, if the direction of the curve $\gamma$ requires the use of an $s$-order Lie bracket, and the approximating curve consists of basic periodic motions in the $\left(x_{1}, x_{2}\right)$ space that are of size $\varepsilon$, then each may at best result in a displacement of order $\varepsilon^{s}$ in the direction of $\gamma$. Hence travel along $\gamma$ for a distance $L$ requires on the order of $L \varepsilon^{1-s}$ repetitions of the basic loop.

The use of sinusoids for nonlinear path planning has a long history. Among the rich literature on this topic, we comment on the relation of this article to a few closely related ones. Much classic work such as [11] generally is restricted to systems of a very special form, e.g. chained (or "differentially flat") systems. Section [III reviews some of these results, and strengthens them by showing that controls of the form $(\sin t, \cos m t)$ annihilate not only the moments of systems in chain form, but indeed all lower order moments. In contrast to the comprehensive work on highly oscillatory controls [12], [13] that develops a general approximation algorithm and general existence results (e.g. for a set of minimally canceling frequencies), this article focuses on explicit formulas using as slowly oscillating controls as possible to achieve the objective and prove that they simultaneously annihilate surprisingly large sets of iterated integral functionals.

From combinatorial-algebraic and functional analytic points of view, one considers a pairing of control inputs 
with iterated integral functionals, which yields system outputs (trajectories $y(\cdot)$ or terminal values $y(T)$ ) [14]. Corresponding to Hall-bases $\mathcal{H}$ for free Lie algebras, dual bases $\left\{\xi_{H}: H \in \mathcal{H}\right\}$ of iterated integral functionals have been identified in, e.g., [15] (coordinates of the second kind, as they appear in exponential product expansions). Alternative bases (coordinates of the first kind $\left\{\zeta_{H}: H \in \mathcal{H}\right\}$, as in a continuous Campbell-Baker-Hausdorff formula) were identified in [16] using a Hopf-algebraic approach. In either case, it is natural quest to find basic sets of controls that are, if not diagonal, at least triangular on these bases. This means that using the graded structures of the Hall bases, one wants controls $u_{H}:[0, T] \mapsto \mathbb{R}^{m}$ such that $\xi_{H}\left(u_{H_{0}}, T\right)=0$ (or $\zeta_{H}\left(u_{H_{0}}, T\right)=0$ ) if $H \prec H_{0}$ and $\xi_{H_{0}}\left(u_{H_{0}}, T\right) \neq 0$ (or $\left.\zeta_{H_{0}}\left(u_{H_{0}}, T\right) \neq 0\right)$. While not yet giving a complete such set, this article identifies explicit formulas for sinusoidal controls that achieves this block triangular structure for a large subset of $\mathcal{H}$. Small illustrative portions of the matrix $\xi_{K}\left(u_{H}\right)$ are shown in the appendix.

The constructions and proof of the main theorem rely on elementary number theory and make critical use of the observation that for nonlinear systems successive integrations split and shift the set of frequencies. This is in fundamental contrast to linear systems theory which is fundamentally built on successive integrations leaving the signal's frequencies unchanged (only modulating their amplitudes). Indeed, in nonlinear systems, for sinusoidal inputs $u_{i}$, all iterated integrals of the controls that appear in the Chen Fliess series are again combinations of polynomials and sinusoids. Indeed, until resonances come into play, the main operation on iterated integrals

$$
R_{u_{c}}: \Upsilon_{w}(u, t) \mapsto \Upsilon_{w c}(u, t)=\int_{0}^{t} \Upsilon_{w}(u, s) u_{c}(s) d s
$$

maps trigonometric polynomials to themselves, however with altered frequencies (this was critically used in [12], [13]).

\section{TECHNICAL BACKGROUND AND TERMINOLOGY}

For higher order iterated integrals and Lie brackets it is convenient to introduce more compact combinatorial notation and symbols. This article only considers two-dimensional distributions, or, correspondingly, nonlinear control systems with controls $u=\left(u_{1}, u_{2}\right)=\left(u_{a}, u_{b}\right)$ taking values in $\mathbb{R}^{2}$. We use the indices $a=1$ and $b=2$ interchangeably, as, depending on the context, either one may be more readable. For the set $Z=\{a, b\}$, called alphabet, let $Z^{*}$ denote the set of all words, that is, finite sequences with values in $Z$, including the empty sequence. Denote concatenation of sequences by juxtaposition, and use standard notation for noncommutative powers $w^{k+1}=w^{k} w$. The length of a word $w$ is denoted by $|w|$ and the sets of words of length equal to $r$ and length less than $r$ are denoted by $Z^{r}$ and $Z^{<r}$. Write $Z^{(i, j)}$ for the set of words $w$ containing $|w|_{a}=i$ and $|w|_{b}=j$ copies of the letters $a$ and $b$, respectively.

The set $Z^{*}$ encodes iterated integral functionals via the map $\Upsilon$ defined by $\Upsilon_{e}(u, t) \equiv 1$ for the empty word $e \in Z^{*}$ and recursively for words $w \in Z^{*}$ and letters $c \in Z$ by

$$
\Upsilon_{w c}(u, t)=\int_{0}^{t} \Upsilon_{w}(u, s) u_{c}(s) d s .
$$

The unique factorization property [17] of Hall bases for the free Lie algebra $L(Z)$ over the set $Z$. allows these to be encoded by words in $Z^{*}$. A possible initial segment of such a Hall set is

$$
\begin{aligned}
& \mathcal{H}=\{a, b, a b, a a b, b a b, a a a b, b a a b, b b a b, a a a a b, \\
& \text { baaab, abaaab, bbaaab, abbaab, babaab, ... }
\end{aligned}
$$

which correspond to the Hall basis of formal brackets

$$
\begin{gathered}
a, b,[a, b],[a,[a, b]],[b,[a, b]],[b,[a,[a, b]]], \ldots \\
\ldots[b,[a,[a, a,[a, b]]]],[[a, b],[a,[a,[a, b]]]], \ldots \\
\ldots[[a, b],[b,[a,[a, b]]]],[[b,[a, b]],[a,[a, b]]] \ldots
\end{gathered}
$$

In analogy with above notation, use the symbols $L^{r}(Z)$, $L^{<r}(Z)$, and $L^{(i, j)}(Z)$ to denote the components of the graded and filtered structures of $L(Z)$, and its finely homogeneous components.

The dual basis for the (Poincaré-Birkhoff-Witt basis) of such Hall basis plays a fundamental role in infinite directed exponential product expansions of the Chen-Fliess series [15], and for minimal realizations of nilpotent systems in terms of cascade polynomial vector fields [14], [16], [18], [19]. For Hall words $H \in \mathcal{H}$ the dual basis elements are distinguished linear combinations of the iterated integral functionals $\Upsilon_{w}$, defined as follows. If $H=c \in \mathcal{H}$ is a letter then $\xi_{c}=\Upsilon_{c}$. If $H=M^{r} K$ is the unique regular factorization of $H$ into Hall words $M$ and $K$ (with $r \geq 1$ maximal), then

$$
\xi_{H}(u, t)=\frac{1}{r !} \int_{0}^{t} \xi_{M}^{r}(u, s) \dot{\xi}_{K}(u, s) d s
$$

where $\dot{\xi}$ denotes the time derivative of $\xi$. For more details on the underlying Zinbiel and Hopf algebra structures see [16].

\section{MULTIPLE FIGURE EIGHT CURVES}

Motivated by the topology of the time-optimal curves for any distribution whose flag has maximal growth vector $r=$ $(2,3,5,6)$ [2], [3], it is natural to first analyze controls $u=$ $\left(u_{1}, u_{2}\right)$, or base curves $\left(x_{1}, x_{2}\right)$, which topologically are generalized figure eight curves with several self-intersections.

If $u:[0, T] \mapsto \mathbb{R}^{2}$ is a continuous control such that $\Upsilon_{w}(u, T)=0$ for all words $w \in Z^{*}$ with $|w|<m$, then, in particular, $\xi_{a^{k} b}(u, T)=0$ for all $k<m-1$.

Nonholonomic systems whose dynamics are entirely determined by these iterated integrals $\Upsilon_{a^{k} b}$ have been studied extensively in the literature under the name of chained systems, see, e.g., [11], [20], and sinusoidal controls have been used effectively for the path planning problem for such systems.

As an introduction, suppose $\Upsilon_{a^{k} b}(u, T)=0$ for all $k<m-1$ and $\Upsilon_{a^{m} b}(u, T) \neq 0$. If in addition $u$ is a continuous control such that $u_{1}$ changes sign only once, then the curve $\left(x_{1}, x_{2}\right)(t)=\left(\Upsilon_{a}(u), \Upsilon_{b}(u)(t)\right.$ must self-intersect in at least $(m-1)$ distinct points. and $u_{2}$ must change sign at least $m-1$ times. Indeed, suppose $u_{a}(t)>0$ for $t \in\left(0, t_{\mathrm{sw}}\right)$ and $u_{a}(t)<0$ for $t \in$ 
$\left(t_{\mathrm{sw}}, T\right), k \geq 1$, and $0 \leq t_{i}<t_{i+1} \leq t_{j}<t_{j+1} \leq$ $T$ are such that $\left(\Upsilon_{a}, \Upsilon_{a^{k} b}\right)\left(u, t_{i}\right)=\left(\Upsilon_{a}, \Upsilon_{a^{k} b}\right)\left(u, t_{j+1}\right)$ and $\left(\Upsilon_{a}, \Upsilon_{a^{k} b}\right)\left(u, t_{i+1}\right)=\left(\Upsilon_{a}, \Upsilon_{a^{k} b}\right)\left(u, t_{j}\right)$. Then there must exist $t^{-} \in\left(t_{i}, t_{i+1}\right)$ and $t^{+} \in\left(t_{j}, t_{j+1}\right)$ such that $\left(\Upsilon_{a}, \Upsilon_{a^{k-1} b}\right)\left(u, t^{-}\right)=\left(\Upsilon_{a}, \Upsilon_{a^{k-1} b}\right)\left(u, t^{+}\right)$. The claim follows inductively, compare figure 1 for an illustration of the (vertically rescaled) graphs of $\left(\Upsilon_{a}, \Upsilon_{a^{k} b}\right)(u)$ for $u=$ $(\sin t, \cos 7 t)$ for $k=0$ on the bottom to $k=7$ on the top.

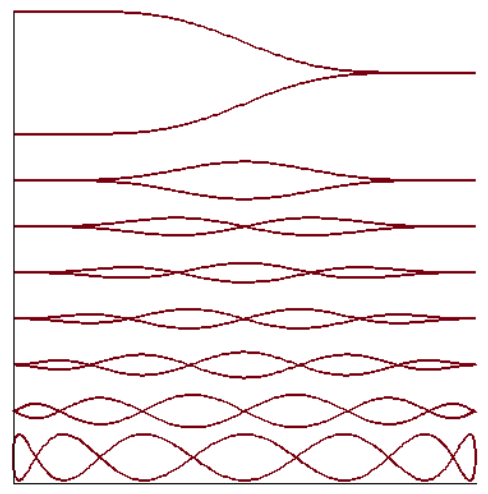

Fig. 1. Moments for curves with frequencies $(1, m)$.

For sinusoids of the form $u=(\sin t, \cos m t)$ whose graphs are Lissajous curves, it is not too hard to inductively find explicit formulas for the associated iterated integrals $\Upsilon_{a^{k} b}(u, t)$. Once the formula is correctly guessed, the proof is immediate using elementary integration techniques.

Lemma 3.1: For $\left.x=\left(x^{a}, x^{b}\right)\right): t \mapsto(1-\cos t, \sin m t)$ and all $t \in \mathbb{R}$, and all $0 \leq k<m \in \mathbb{Z}^{+}$

$$
\Upsilon_{a^{k} b}(u, t)=\sum_{i=0}^{k}\left(\begin{array}{l}
k \\
i
\end{array}\right) \frac{(-)^{k}(m-k+i-1) !}{2^{k}(m+i) !} \sin (m-k+2 i) t .
$$

For illustration, for $u=(\sin t, \cos 7 t)$ the first moments (that appear in chained systems) are

$$
\begin{aligned}
\Upsilon_{b}(u, t) & =\frac{1}{7} \sin 7 t \\
\Upsilon_{a b}(u, t) & =\frac{1}{84} \sin 6 t-\frac{1}{112} \sin 8 t \\
\Upsilon_{a^{2} b}(u, t) & =\frac{\sin 5 t}{840}-\frac{\sin 7 t}{672}+\frac{\sin 9 t}{2,016} \\
\Upsilon_{a^{3} b}(u, t) & =\frac{\sin 4 t}{6,720}-\frac{\sin 6 t}{4,480}+\frac{\sin 8 t}{8,064}-\frac{\sin 10 t}{40,320} \\
\Upsilon_{a^{7} b}(u, 2 \pi) & =\frac{\pi}{322,560} .
\end{aligned}
$$

The mechanism responsible for the pattern of frequencies that appear with nonzero amplitudes in these integrals (that are familiar from systems in chain form) is apparent. But we prove more: The same controls annihilateall iterated integrals corresponding to all words of length at most $m$, not only these of form $a^{k} b$, or $w$ with $|w|_{b}=1$.

Proposition 3.2: Suppose $m \geq 1$ is a positive integer and $u:[0,2 \pi] \mapsto \mathbb{R}^{2}$ is defined by $u(t)=(\sin t, \cos m t)$. Then for all $k<m$ and all words $w \in Z^{*}(a, b)$, if $|w|<m$ then $\Upsilon_{w}(u, 2 \pi)=0$, and $\xi_{a^{m} b}(u, 2 \pi) \neq 0$.

Indeed this proposition is a special case of the main theorem in the next section, and its direct proof follows the same pattern. This result is stated separately as it was directly motivated from the original question about optimal approximations of inadmissible curves for distributions with maximal growth vector $r=(2,3,5, \ldots N-1, N)$, and it is intimately related to the previously studied systems in chain form.

\section{GENERAL LISSAJOUS CURVES}

Proposition 3.2 and its original proof motivate one to consider more general controls defined by sinusoids. Generically for a distribution spanned by vector fields $f_{1}$ and $f_{2}$ with maximal growth vector $r=\left(r_{1}, \ldots, r_{s-1}, r_{s}\right)=$ $(2,3,5, \ldots N-1, N)$ each of the iterated Lie brackets in $L^{s}\left(f_{1}, f_{2}\right)$ of length $s$ will be linearly independent from $\Delta^{[s-1]}$ at every point in some open neighborhood of the curve under consideration. For such distributions it suffices to find controls, as above, for which $\Upsilon_{w}(u, T)=0$ for all $w$ with $|w|<s$ and $\Upsilon_{w_{0}}(u, T) \neq 0$ for one $w_{0}$ with $\left|w_{0}\right|=s$.

The next more ambitious task is to find $u$ such that $\Upsilon_{w}(u, T) \neq 0$ for $w \in Z^{(i, j)}$ for a fixed finely homogeneous component such that $i+j=s+1$. Even further, one may demand that $\xi_{H_{0}}(u, T) \neq 0$ for a specific $H_{0} \in \mathcal{H}^{(i, j)}$, but $\xi_{H}(u, T)=0$ for all other $H \in \mathcal{H}^{i+j} \backslash\left\{H_{0}\right\}$. This section provides explicit formulas for controls $u$ that achieve the first of these two objectives for a large set of homogeneous components $Z^{(i, j)}$. The second objective is subject of current research and requires substantially more attention to combinatorial structure of Hall bases and the Zinbiel structure of their associated iterated integral functionals, compare also the classic effort [21].

First recall that the Chen integrals $\Upsilon_{w}(u, T)$ are invariants of the curve $x(\cdot)$, and, in particular, invariant under reparameterizations of time [22]. From both the geometric analytic point of view it is natural to work with the absolutely continuous base curve $\left(x_{1}, x_{2}\right)$ defined by the primitive of the controls $\left(u_{1}, u_{2}\right)=\left(\dot{x}_{1}, \dot{x}_{2}\right)$. The proposed construction makes use of Lissajous curves that are determined up to reparameterization and rescaling, by choices of $u_{i} \in$ $\left\{\sin m t: m \in \mathbb{Z}^{+}\right\} \cup\left\{\cos m t: m \in \mathbb{Z}^{+}\right\}$. In order for the integrals $\Upsilon_{w}(u, 2 \pi)$ and $\xi_{H}(u, 2 \pi)$ to not all be trivial, the controls have to be chosen such that the base curve $\left(x_{1}, x_{2}\right)$ not trace back and fourth along the same curve. Up to rotations, reflections, time-shift, and reparameterizations, all nontrivial Lissajous curves in the plane may be obtained by choosing $\left(x_{1}, x_{2}\right)(t)=(\cos m t, \sin n t)$ with $m$ and $n$ relatively prime positive integers and $m$ odd. For purposes of brevity of this conference article we state the theorem for only a restricted class Lissajous curves, but note that after rotation, by, possibly fractional, multiples of $\frac{\pi}{2}$ and other symmetry operations one easily derives corresponding results for other finely homogeneous sets of words. Due to the graded structure of the space of iterated integral functionals, it is straightforward to rescale, and adjust for the usual constraint $u_{1}^{2}+u_{2}^{2}=1$.

Theorem 4.1: Suppose $m$ and $n$ are relatively prime positive integers, and $u:[0,2 \pi] \mapsto \mathbb{R}^{2}$ is defined by $u(t)=$ $(\sin m t, \cos n t)$ if $m$ is odd, and $u(t)=(\cos m t, \cos n t)$ if $m$ is even. Then $\Upsilon_{w}(u, T)=0$ for all $w \in Z^{\leq(m+n)} \backslash Z^{(m, n)}$, 
but $\Upsilon_{w_{0}}(u, T) \neq 0$ for some $w_{0} \in Z^{(m, n)}$. Thus also $\xi_{H}(u, T)=0$ for all Hall words $H \in \mathcal{H}^{\leq(m+n)} \backslash \mathcal{H}^{(m, n)}$, but $\xi_{H_{0}}(u, T)=0$ for some $H_{0} \in \mathcal{H}^{(m, n)}$.

Remark 4.2: To generate motion in directions of iterated Lie brackets corresponding to Hall words $H \in \mathcal{H}^{(i, j)}$ with $(i, j)$ not relatively prime, the above distinguished sinusoidal controls may be combined with traditional schemes. E.g., using $u_{1}=C_{2} S_{1}=(\cos 2 t, \sin t)$ and $u_{2}=S_{1} C_{2}=$ $(\sin t, \cos 2 t)$ (both on $[0,2 \pi])$ generate motions in the directions of $\left[f_{1},\left[f_{1}, f_{2}\right]\right]$ and $\left[f_{2},\left[f_{1}, f_{2}\right]\right]$, respectively, their concatenation $u^{a a b b a b}=C_{2} S_{1} \# S_{1} C_{2} \#\left(C_{2} S_{1}\right)^{-} \#\left(S_{1} C_{2}\right)^{-}$ (with $u^{-}$indicating the time reversed input $u^{-}(t)=$ $u(2 \pi-t))$, generates nonzero motion in the direction of $\left[\left[f_{2},\left[f_{1}, f_{2}\right]\right],\left[f_{1},\left[f_{1}, f_{2}\right]\right]\right]$ corresponding to the Hall word $H_{0}=b a b a a b \in \mathcal{H}^{(3,3)}$, and, in particular, $\left.\xi_{H}\left(u^{H_{0}}, 2 \pi\right)\right)=0$ for all $H \in \mathcal{H}^{\leq 7} \backslash \mathcal{H}^{(3,3)}$. The 7 is not a type-oh. This input is not as efficient as the sinusoidal controls for $\operatorname{gcd}(i, j)=1$, but it is still far less complex than a naive approach that does not take advantage of theorem 4.1

Proof. We present an outline of the proof for the case of $m$ is even, and $u(t)=(\cos m t, \cos n t)$. The arguments for the more general case of $m$ odd and $u(t)=(\sin m t, \cos n t)$ are very similar but longer. They, and a more detailed proof will be given in a longer journal article.

For notational ease represent subspaces of the space of trigonometric polynomials by pairs of sets integers. For finite sets $S_{1}, S_{2} \subseteq \mathbb{Z}$, define the map $\mathcal{T}: 2_{\text {fin }}^{\mathbb{Z}} \times 2_{\text {fin }}^{\mathbb{Z}} \mapsto$ $C([0,2 \pi], \mathbb{R})$ by

$$
\mathcal{T}\left(\left[S_{1}, S_{2}\right]\right)=\left\{\sum_{i \in S_{1}} a_{i}(1-\cos i t)+\sum_{j \in S_{2}} b_{j} \sin j t: a_{i}, b_{j} \in \mathbb{R}\right\}
$$

Conversely, for any trigonometric polynomial function $p: t \mapsto \sum_{|i|<M} a_{i}(1-\cos i t)+b_{i} \sin i t$ let $\mathcal{F}(p)$ be the pair of sets of frequencies with nonzero amplitudes. More precisely, if $\mathcal{F}(p)=\left[S_{1}, S_{2}\right]$ then $0 \in S_{1}$ if and only if $p(0) \neq 0,0 \neq i \in S_{1}$ if and only if $\int_{0}^{2 \pi} p(t) \cos i t d t \neq 0$ and $j \in S_{2}$ if and only if $\int_{0}^{2 \pi} p(t) \sin j t d t \neq 0$. Note that $\mathcal{T}$ is a left inverse of $\mathcal{F}$, but not a right inverse (since we have chosen $\mathcal{T}(S)$ as a subspace of $C([0,2 \pi]))$.

For sinusoidal controls $u_{c}(t)=\cos i t$ or $u_{c}(t)=\sin j t$, the associated integral operators as in (3)

$$
\begin{aligned}
& \left(\tilde{I}_{j}^{+} f\right)(t)=\int_{0}^{t} f(s) \cos j s d s, \quad \text { and } \\
& \left(\tilde{I}_{j}^{-} f\right)(t)=\int_{0}^{t} f(s) \sin j s d s,
\end{aligned}
$$

when restricted to trigonometric polynomials $f$, induce operations on the sets of frequencies. Using the shorthand notation $S \pm j=\{i+j: i \in S\} \cup\{i-j: i \in S\}$, for any finite subsets $S_{1}, S_{2} \subseteq \mathbb{Z}$, define (with no special consideration of whether $0, \pm j \in S_{1}, S_{2}$ )

$$
\begin{aligned}
I_{j}^{+}\left(\left[S_{1}, S_{2}\right]\right. & =\left[S_{1} \pm j, S_{2} \pm j\right] \text { and } \\
I_{j}^{-}\left(\left[S_{1}, S_{2}\right]\right. & =\left[S_{2} \pm j, S_{1} \pm j\right] .
\end{aligned}
$$

In general, $\tilde{I}_{i}^{ \pm}$and $\tilde{I}_{j}^{ \pm}$do not commute. But the induced operators on sets of frequencies do: For all $i, j \in \mathbb{Z}^{+}$

$$
I_{i}^{+} I_{j}^{+}=I_{j}^{+} I_{i}^{+}, I_{i}^{+} I_{j}^{-}=I_{j}^{-} I_{i}^{+}, I_{i}^{-} I_{j}^{-}=I_{j}^{-} I_{i}^{-} .
$$

Using trigonometric identities such as (for $|i| \neq|j|$ )

$$
\int_{0}^{t} \sin i s \sin j s d s=\frac{\sin (i-j) t}{2(i-j)}-\frac{\sin (i+j) t}{2(i+j)}
$$

one readily proves the following commutation relations.

Lemma 4.3: If $j \in \mathbb{Z} \backslash\{0\}$ and $S=\left[S_{1}, S_{2}\right]$ is a pair of finite subsets of $\mathbb{Z}$, then

$$
\begin{aligned}
\tilde{I}_{j}^{+} \circ \mathcal{T}(S) & \subseteq \mathcal{T} \circ I_{j}^{+}(S) \text { if } j \notin S_{1}, \text { and } \\
\tilde{I}_{j}^{-} \circ \mathcal{T}(S) & \subseteq \mathcal{T} \circ I_{j}^{-}(S) \text { if } j \notin S_{2} .
\end{aligned}
$$

On the other hand, if $p \in \mathcal{T}\left(\left[S_{1}, S_{2}\right]\right)$ and $j \in S_{1}$ or $-j \in S_{1}$ (or $j \in S_{2}$ or $-j \in S_{2}$ ), then $\tilde{I}_{j}^{+}(p)(2 \pi) \neq 0$ (respectively, $\left.\tilde{I}_{j}^{-}(p)(2 \pi) \neq 0\right)$.

With these technical terms in place, suppose $m, n$ are relatively prime positive integers and $u(t)=(\cos m t, \cos n t)$ Then from $\Upsilon_{a}(u, 2 \pi)=\Upsilon_{b}(u, 2 \pi)=0$ it readily follows that $\Upsilon_{a^{k}}(u, 2 \pi)=\Upsilon_{b^{k}}(u, 2 \pi)=0$ for all $k \geq 1$. Next, suppose $r, s \geq 1$ and fix any word $w \in Z^{(r, s)}$, and consider $\left[S_{1}, S_{2}\right]=\mathcal{F} \circ \Upsilon_{w}(u)$. Using the properties of $I^{+}$, if $r+s$ is odd then $S_{1}=\emptyset$ and

$$
S_{2} \subseteq\{(r-2 i) m+(s-2 j) n: 0 \leq i \leq m, 0 \leq j \leq n\} .
$$

If $r+s$ is even, then $S_{2}=\emptyset$ and

$$
S_{1} \subseteq\{(r-2 i) m+(s-2 j) n: 0 \leq i \leq m, 0 \leq j \leq n\} .
$$

Using that $m$ and $n$ are relatively prime, it follows that if $r \leq n$ and $s \leq m$ but $r+s \neq m+n$ then $0 \notin$ $S_{2}$ and $0 \notin S_{1}$ in each of the two cases. Consequently $\Upsilon_{w}(u, 2 \pi)=0$. On the other hand, if $r=m$ and $s=n$, then $\Upsilon_{b^{m-1} a^{n} b}((\cos m t, \cos n t), 2 \pi) \neq 0$, and hence also $\xi_{b^{m-1} a^{n} b}((\cos m t, \cos n t), 2 \pi) \neq 0$.

Tables that illustrate how low order Lissajous curves are block-triangular on high-order iterated integral functionals corresponding to dual Hall bases are given in the appendix.

\section{ONE SELF-INTERSECTION SUFFICES}

The isoperimetric problem, translated into this framework, is modeled by the three dimensional control system $\dot{x}_{1}=u_{1}, \dot{x}_{2}=u_{2}, \dot{y}=\left(x_{1} u_{2}-x_{2} u_{1}\right)$ The objective is, for given $T>0$, to maximize $y(T)$ subject to the constraints $x_{i}(0)=x_{i}(T)=y(0)=0$ and $u_{1}^{2}+u_{2}^{2} \equiv 1$. Up to reparameterization, the well-known solution is $u=$ $(\cos (2 \pi t / T), \sin (2 \pi t / T))$. For the next larger system, e.g., the $\left(x_{1}, x_{2}, y, z_{1}\right)$-subsystem of (15), the additional condition that $y(T)=0$ requires (via an elementary application of Green's theorem) that the curve $\left(x_{1}, x_{2}\right):[0, T] \mapsto \mathbb{R}^{2}$ to not be injective, i.e., it must selfintersect nontrivially in order for $\left.z_{(} T\right) \neq z_{1}(0)$. In [2] it is shown that the optimal curves for the next larger system have two points of selfintersection and topologically are equivalent to the double figure eight, or the curve $(\cos t, \sin 3 t)$. Together with the examples discussed in section III one may conjecture that, in order to render ever higher moments simultaneously equal to zero, the base curve must have increasing numbers of self intersections. But contrary to the case discussed in section III we show that without the restriction that $u_{1}$ changes sign only once, it is possible to render the next 
moments simultaneously equal to zero without any further self-intersections.

We consider the following system in normal form for the sub-Riemannian problem with growth vector $r=(2,3,5,6)$.

$$
\begin{aligned}
\dot{x}_{1} & =u_{1} \\
\dot{x}_{2} & =u_{2} \\
\dot{y} & =x_{1} u_{2}-x_{2} u_{1} \\
\dot{z}_{1} & =\left(x_{1} u_{2}-x_{2} u_{1}\right) x_{1} \\
\dot{z}_{2} & =\left(x_{1} u_{2}-x_{2} u_{1}\right) x_{2} \\
\dot{w} & =\left(x_{1} u_{2}-x_{2} u_{1}\right)\left(x_{2}^{2}-x_{1}^{2}\right)
\end{aligned}
$$

Proposition 5.1: There exists an absolutely continuous closed curve $\left(x_{1}, x_{2}\right):[0, T] \mapsto \mathbb{R}^{2}$ that has only a single point of self-intersection such that the corresponding lifted curve defined by system (15) satisfies (for zero initial data) $\left(x_{1}, x_{2}, y, z_{1}, z_{2}\right)(T)=0$ and $w(T) \neq 0$.

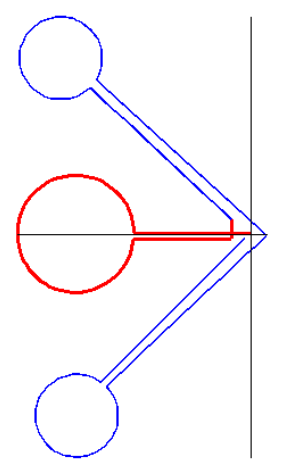

Fig. 2. $\varepsilon$-exploded view of triple lollipop. First middle, then top loop.

Proof outline. A possible solution is sketched in figure 2 For a start consider the collapsed view of this curve with parallel line segments collapsed in pairs and traversed back and forth. The collapsed curve consists of line segments emanating from the origin along the first axis and at $45^{\circ}$ angles to it, and which that connect with circles with radii 1 and $\sqrt{2} / 2$ that are centered at $(-2,0)$ and at $\left(-\frac{5}{2}, \pm \frac{5}{2}\right)$, respectively.

It is a lengthy, but elementary, computation to verify that this curve satisfies the desired condition for $\left(x_{1}, x_{2}, y, z_{1}, z_{2}, w\right)(T)$. The graphs of these coordinates as functions of time are illustrated in figure 3 .

Using the controllability of the system about this curve, it is not hard to argue that one can deform the curve by an arbitrarily small amount so that still $\left(x_{1}, x_{2}, y, z_{1}, z_{2}\right)(T)=$ 0 and $w(T) \neq 0$, and at the same time there exists a single pair of points $t_{1}, t_{2} \in[0,2 \pi)$ such that $\left(x_{1}, x_{2}\right)\left(t_{1}\right)=$ $\left(x_{1}, x_{2}\right)\left(t_{2}\right)$, as shown in figure 2

\section{CONCLUSIONS AND FUTURE WORKS}

\section{A. Conclusions}

This article exhibits very simple, low frequency sinusoidal control that are shown to annihilate a large number of iterated integral functionals and selectively excite only narrowly specified modes corresponding to high order iterated Lie

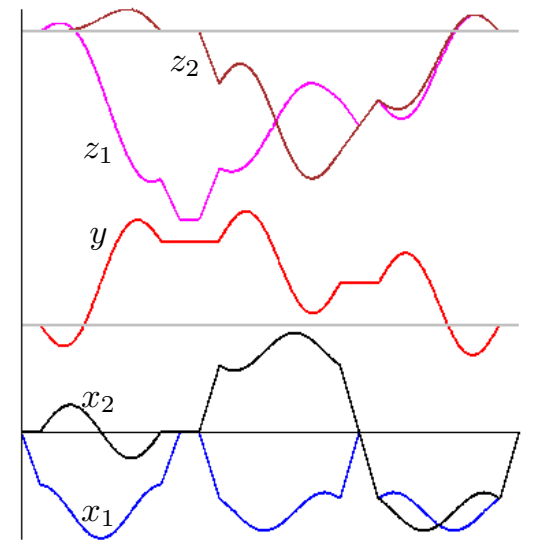

Fig. 3. Moments of the triple lollipop as functions of time.

brackets. These controls promise to improve path planning designs for nonholonomic systems. It is also shown that the number of self-intersections is not an appropriate measure to fine-tune the quantification of the complexity of controls required to achieve motion in the direction of increasingly higher order Lie brackets.

\section{B. Future Work}

Starting with iterated Lie brackets of length five the dimensions of the finely homogeneous subspaces of the free Lie algebra are generally larger than one. Finding enough distinct curves that excite each direction separately corresponds to the ultimate goal of presenting complete sets of controls which are triangular on the set of iterated integral functionals that are dual to a basis for the free Lie algebra. Such a set of explicitly given controls would significantly facilitate the algorithmic solution of path planning problems with much simpler controls that have been used in the past.

The next steps are to systematically identify whether e.g. rotations can provide enough different directions, and to systematically design basic loops that correspond to iterated integral functionals for Hall words in finely homogeneous components whose indices are not relatively prime. The next more challenging combinatorial problem is to extend this study to $k$-distributions with $k \geq 3$.

\section{ACKNOWLEDGMENTS}

The first author expresses his gratitude to the Universite de Toulon for its hospitality during his stay in summer 2013.

\section{REFERENCES}

[1] G. Lafferriere and H. J. Sussmann, "Motion planning for controllable systems without drift," in Proceedings IEEE Conference on Robotics and Automation, Sacramento, CA, 1991, pp. 1148-1153.

[2] N. Boizot and J.-P. Gauthier, "On the motion planning of the ball with a trailer," Math. Control Relat. Fields, vol. 3, 2013.

[3] _ - "Motion planning for kinematic systems," IEEE Trans. Automat. Control, vol. 58, no. 6, pp. 1430-1442, 2013.

[4] C. Romero-Meléndez, J. P. Gauthier, and F. Monroy-Pérez, "On complexity and motion planning for co-rank one sub-Riemannian metrics," ESAIM Control Optim. Calc. Var., vol. 10, no. 4, pp. 634 655, 2004. 
[5] A. A. Agrachev and Y. L. Sachkov, Control theory from the geometric viewpoint, ser. Encyclopaedia of Mathematical Sciences. Berlin: Springer-Verlag, 2004, vol. 87, control Theory and Optimization, II.

[6] F. Jean, "Complexity of nonholonomic motion planning," Internat. J. Control, vol. 74, no. 8, pp. 776-782, 2001.

[7] — , "Entropy and complexity of a path in sub-Riemannian geometry," ESAIM Control Optim. Calc. Var., vol. 9, pp. 485-508 (electronic), 2003.

[8] J.-P. Gauthier and V. Zakalyukin, "Nonholonomic interpolation for kinematic problems, entropy and complexity," in Mathematical control theory and finance. Berlin: Springer, 2008, pp. 187-210.

[9] — - "On the motion planning problem, complexity, entropy, and nonholonomic interpolation," J. Dyn. Control Syst., vol. 12, no. 3 , pp. 371-404, 2006.

[10] J.-P. Gauthier, B. Jakubczyk, and V. Zakalyukin, "Motion planning and fastly oscillating controls," SIAM J. Control Optim., vol. 48, no. 5, pp. 3433-3448, 2009/10.

[11] R. M. Murray and S. S. Sastry, "Nonholonomic motion planning: steering using sinusoids," IEEE Trans. Automat. Control, vol. 38, no. 5, pp. 700-716, 1993.

[12] W. Liu, "An approximation algorithm for nonholonomic systems," SIAM J. Control Optim., vol. 35, no. 4, pp. 1328-1365, 1997.

[13] H. J. Sussmann and L. Wensheng, "Limits of highly oscillatory controls and the approximation of general paths by admissible trajectories," in Proceedings IEEE Conference Decision and Control, Brighton, England, 1991, pp. 437-442.

[14] M. Kawski and H. J. Sussmann, "Noncommutative power series and formal Lie-algebraic techniques in nonlinear control theory," in Operators, systems, and linear algebra, ser. European Consort. Math. Indust. Stuttgart: Teubner, 1997, pp. 111-128.

[15] H. J. Sussmann, "A product expansion for the Chen series," in Theory and applications of nonlinear control systems (Stockholm, 1985). Amsterdam: North-Holland, 1986, pp. 323-335.

[16] E. Gehrig and M. Kawski, "A hopf-algebraic formula for compositions of noncommuting flows," in Proceedings IEEE Conference Decision and Control, Cancun, Mexico, 2008, pp. 156-1574.

[17] G. Viennot, Algèbres de Lie libres et monoüdes libres, ser. Lecture Notes in Mathematics. Berlin: Springer, 1978, vol. 691, bases des algèbres de Lie libres et factorisations des monoïdes libres.

[18] M. Kavski, "Chronological algebras: combinatorics and control," in Geometric control theory (Russian) (Moscow, 1998), ser. Itogi Nauki Tekh. Ser. Sovrem. Mat. Prilozh. Temat. Obz. Moscow: Vseross. Inst. Nauchn. i Tekhn. Inform. (VINITI), 1999, vol. 64, pp. 144-178.

[19] M. Kawski, "The combinatorics of nonlinear controllability and noncommuting flows," in Mathematical control theory, Part 1, 2 (Trieste, 2001), ser. ICTP Lect. Notes, VIII. Abdus Salam Int. Cent. Theoret. Phys., Trieste, 2002, pp. 223-311.

[20] D. Tilbury, R. M. Murray, and S. S. Sastry, "Trajectory generation for the $n$-trailer problem using Goursat normal form," IEEE Trans. Automat. Control, vol. 40, no. 5, pp. 802-819, 1995.

[21] H. J. Sussmann and L. Wensheng, "Lie bracket extensions and averaging: the single-bracket case," in Nonholonomic Motion Planning, Z. X. Li and J. F. Canny, Eds. Boston: Kluwer, 1993, pp. 109-148.

[22] K.-T. Chen, "Integration of paths, geometric invariants and a generalized Baker-Hausdorff formula," Ann. of Math. (2), vol. 65, pp. 163-178, 1957.

\section{APPENDIX}

The tables on the right list the values of $\xi_{H}(u, 2 \pi)$ for selected Lissajous curves whose relatively prime frequencies sum to 6 and 7, respectively. The rows are indexed by a Hall basis for the initial nilpotent section $L^{\leq 7}(\{a, b\})$ of the free Lie algebra $L(\{a, b\})$. The column headers $S_{m, n}$ and $C_{m, n}$ correspond to the primitives $\left(x_{1}, x_{2}\right)=(\sin m t, \sin n t)$ and $\left(x_{1}, x_{2}\right)=(\sin m t, 1-\cos n t)$ of the controls, respectively. The table entries are values of $\xi_{H}$ (up to rescaling by powers of $m$ and $n$ and multi-factorials).

Apparent is the block triangular structure of the matrix, illustrating $\xi_{H}(\cos m t, \cos n t)=0$ and $\xi_{H}(\cos m t, \sin n t)=$ 0 if $|H|<m+n$. Moreover, these entries are also zero if $|H|=m+n$, but $H \notin \mathcal{H}^{(m, n)}$.
TABLE I

HALL MOMENTS OF ORDER AT MOST 6

$\begin{array}{ccccccc} & S_{1,4} & S_{2,3} & S_{3,2} & S_{4,1} & C_{1,5} & C_{5,1} \\ a & 0 & 0 & 0 & 0 & 0 & 0 \\ b & 0 & 0 & 0 & 0 & 0 & 0 \\ {[a b]} & 0 & 0 & 0 & 0 & 0 & 0 \\ {[a[a b]]} & 0 & 0 & 0 & 0 & 0 & 0 \\ {[b[a b]]} & 0 & 0 & 0 & 0 & 0 & 0 \\ {[a[a[a b]]]} & 0 & 0 & 0 & 0 & 0 & 0 \\ {[b[a[a b]]]} & 0 & 0 & 0 & 0 & 0 & 0 \\ {[b[b[a b]]]} & 0 & 0 & 0 & 0 & 0 & 0 \\ {[a[a[a[a b]]]]} & \frac{\pi}{2} & 0 & 0 & 0 & 0 & 0 \\ {[b[a[a[a b]]]]} & 0 & -\frac{3 \pi}{8} & 0 & 0 & 0 & 0 \\ {[[a b][a[a b]]]} & 0 & -\frac{9 \pi}{8} & 0 & 0 & 0 & 0 \\ {[[a b][b[a b]]]} & 0 & 0 & -\frac{\pi}{2} & 0 & 0 & 0 \\ {[b[b[a[a b]]]]} & 0 & 0 & \frac{\pi}{4} & 0 & 0 & 0 \\ {[b[b[b[a b]]]]} & 0 & 0 & 0 & -\frac{\pi}{8} & 0 & 0 \\ {[a[a[a[a[a b]]]]]} & 0 & 0 & 0 & 0 & \frac{5 \pi}{16} & 0 \\ {[b[a[a[a[a b]]]]]} & 0 & 0 & 0 & 0 & 0 & 0 \\ {[[a b][a[a[a b]]]]} & 0 & 0 & 0 & 0 & 0 & 0 \\ {[b[b[a[a[a b]]]]]} & 0 & 0 & 0 & 0 & 0 & 0 \\ {[[a b][b[a[a b]]]]} & 0 & 0 & 0 & 0 & 0 & 0 \\ {[[a[a b]][b[a b]]]} & 0 & 0 & 0 & 0 & 0 & 0 \\ {[[a b][b[b[a b]]]]} & 0 & 0 & 0 & 0 & 0 & 0 \\ {[b[b[b[a[a b]]]]]} & 0 & 0 & 0 & 0 & 0 & 0 \\ {[b[b[b[b[a b]]]]]} & 0 & 0 & 0 & 0 & 0 & \frac{\pi}{16}\end{array}$

TABLE II

HALL MOMENTS OF ORDER 7

$\begin{array}{lcccccc} & S_{1,6} & S_{2,5} & S_{3,4} & S_{4,3} & S_{5,2} & S_{6,1} \\ {[a[a[a[a[a[a b]]]]]]} & -\frac{3 \pi}{16} & 0 & 0 & 0 & 0 & 0 \\ {[b[a[a[a[a[a b]]]]]} & 0 & \frac{5 \pi}{32} & 0 & 0 & 0 & 0 \\ {[[a b][a[a[a[a b]]]]]} & 0 & \frac{25 \pi}{96} & 0 & 0 & 0 & 0 \\ {[[a[a b]][a[a[a b]]]]} & 0 & \frac{25 \pi}{32} & 0 & 0 & 0 & 0 \\ {[b[b[a[a[a[a b]]]]]]} & 0 & 0 & -\frac{\pi}{8} & 0 & 0 & 0 \\ {[[a b][b[a[a[a b]]]]]} & 0 & 0 & -\frac{\pi}{2} & 0 & 0 & 0 \\ {[[a b][[a b][a[a b]]]]} & 0 & 0 & -2 \pi & 0 & 0 & 0 \\ {[[b[a b]][a[a[a b]]]]} & 0 & 0 & -\frac{\pi}{10} & 0 & 0 & 0 \\ {[[a[a b]][b[a[a b]]]]} & 0 & 0 & \frac{\pi}{4} & 0 & 0 & 0 \\ {[[a[a b]][b[b[a b]]]]} & 0 & 0 & 0 & -\frac{9 \pi}{160} & 0 & 0 \\ {[[b[a b]][b[a[a b]]]]} & 0 & 0 & 0 & \frac{9 \pi}{64} & 0 & 0 \\ {[[a b][[a b][b[a b]]]]} & 0 & 0 & 0 & \frac{27 \pi}{32} & 0 & 0 \\ {[[a b][b[b[a[a b]]]]]} & 0 & 0 & 0 & -\frac{9 \pi}{32} & 0 & 0 \\ {[b[b[b[a[a[a b]]]]]]} & 0 & 0 & 0 & \frac{3 \pi}{32} & 0 & 0 \\ {[[b[a b]][b[b[a b]]]]} & 0 & 0 & 0 & 0 & \frac{\pi}{8} & 0 \\ {[[a b][b[b[b[a b]]]]]} & 0 & 0 & 0 & 0 & \frac{\pi}{24} & 0 \\ {[b[b[b[b[a[a b]]]]]]} & 0 & 0 & 0 & 0 & -\frac{\pi}{16} & 0 \\ {[b[b[b[b[b[a b]]]]]]} & 0 & 0 & 0 & 0 & 0 & \frac{\pi}{32}\end{array}$

Additional columns corresponding to symmetries such as rotations of the frame (or, correspondingly of the control) increase the rank of the matrix. But at this time the authors do not know whether it is possible to obtain full rank block triangular matrices for all orders $(m+n)$ using only lowfrequency Lissajous curves as inputs. 